\title{
Germanica
}

\section{La communauté et la mort : la culture allemande devant la première guerre mondiale}

Gemeinschaft und Tod: die deutsche Kultur und der Erste Weltkrieg

\section{Domenico Losurdo}

\section{(2) OpenEdition \\ Journals}

Édition électronique

URL : http://journals.openedition.org/germanica/2427

DOI : $10.4000 /$ germanica.2427

ISSN : 2107-0784

Éditeur

Université de Lille

Édition imprimée

Date de publication : 31 décembre 1990

Pagination : 29-51

ISBN : 9782913857025

ISSN : 0984-2632

Référence électronique

Domenico Losurdo, «La communauté et la mort : la culture allemande devant la première guerre mondiale », Germanica [En ligne], 8| 1990, mis en ligne le 25 novembre 2014, consulté le 06 octobre 2020. URL: http://journals.openedition.org/germanica/2427 ; DOI : https://doi.org/10.4000/ germanica.2427

Ce document a été généré automatiquement le 6 octobre 2020 .

(C) Tous droits réservés 


\title{
La communauté et la mort : la culture allemande devant la première guerre mondiale
}

\author{
Gemeinschaft und Tod: die deutsche Kultur und der Erste Weltkrieg
}

Domenico Losurdo

L'éclatement de la première guerre mondiale est vécu par un grand nombre d'intellectuels européens comme une nouvelle preuve de la crise irréversible non seulement du matérialisme historique, mais aussi de toute «façon unilatéralement naturaliste de penser et de sentir " - l'expression, comme nous le verrons, est de Husserl. L'approche économique et matérielle du monde historique révélait sa banqueroute devant un conflit qui se configurait, selon de nombreuses publications, comme le heurt d'idéaux et de visions du monde opposés, et même comme une guerre de religion et de foi, un Glaubenskrieg ${ }^{1}$. Quel sens cela avait-il que de continuer à parler de luttes de classes ou même seulement d'intérêts matériels devant un conflit qui semblait transcender toute dimension matérielle et démontrer la supériorité du spirituel sur l'économique, et devant l'expérience d'une communauté nationale merveilleusement et intimement unie à l'heure du danger et de la guerre? Il s'agit d'une expérience qu'il n'est pas rare de voir déborder dans le mystique. Stefan Zweig nous a laissé une description fort efficace du climat des journées qui ont immédiatement suivi l'éclatement de la guerre. Nous sommes à Vienne: «Des centaines de milliers de personnes sentaient alors comme jamais ce qu'elles auraient dû sentir en temps de paix : c'est-à-dire qu'elles appartenaient à une grande nation [...]. Chaque individu était appelé à jeter dans la grande masse ardente son moi petit et mesquin pour se purifier de tout égoïsme. Toutes les différences de classe, de langue, de religion, étaient, en ce moment grandiose, submergées par le grand courant de la fraternité [...]. Chaque individu assistait à un élargissement de son propre moi, c'est-àdire qu'il n'était plus une personne isolée, mais se savait inséré dans une masse, faisait partie d'un peuple, et sa personne négligeable avait acquis une raison d'être $»^{2}$. À ce moment-là, nous sommes bien loin du climat de « déclin de l'Occident » ou de l'Europe 
qui se répandra par la suite; dans le ravissement des premiers jours ou des premiers mois de guerre, ce qui a décliné, ce n'est pas seulement le matérialisme historique, mais plus généralement toute vision du monde vulgaire et prosaïque, donc incapable de comprendre et d'apprécier l'expérience extraordinaire de l'unité et de la plénitude spirituelle qui s'est réalisée à l'intérieur de chacune des nations de l'Occident, bien qu'engagées dans une lutte mortelle l'une contre l'autre.

2 Même pour Max Weber, pourtant bien éloigné, évidemment, de tout "spiritualisme ", le plus éthéré comme le plus agité, cela n'a pas de sens que de vouloir expliquer la guerre par la collision entre des « intérêts économiques » opposés; naturellement sont en jeu également des intérêts de puissance, mais ceux-ci, bien loin d'être réductibles à la sphère économique, révèlent une dimension culturelle et spirituelle profonde. Et de toute façon, au moins en ce qui concerne l'Allemagne, elle est entrée en guerre, même au risque de la perdre, en écoutant la voix du "destin », en défense de son « honneur " 3. La mesquine comptabilité économique peut servir peut-être à expliquer les vues des Français, mais ajoute M. Weber, «celui qui parmi nous pourrait avoir un objectif de guerre de ce genre, ne serait pas allemand; l'existence allemande est notre objectif de guerre, pas le profit " 4 . La guerre se présente alors à plus forte raison comme "vraiment grande et merveilleuse au-delà de toute attente ». Groß und wunderbar: c'est une expression qui est constamment reprise, et c'est cela la guerre, selon Weber, indépendamment du résultat final ${ }^{5}$. Ce n'est pas l'expansion de l'Allemagne, le résultat le plus important et le plus durable. D'autres valeurs sont en jeu, et plus hautes. Pour les comprendre, il est bon de faire intervenir le témoignage de Marianne Weber, qui décrit ainsi l'expérience de son mari dans le travail de direction d'un hôpital militaire dans le district de Heidelberg. Il s'agit de soigner et d'assister ceux qui « se sont donnés sans réserve à l'Entier» (dem Ganzen). Et autour de ce devoir s'établit une unité chorale sans précédent: "Comme ils sont merveilleux, ces premiers mois! La vie intérieure entière est reconduite à des lignes simples, grandes et communautaires. Tout ce qui est sans importance s'évanouit. Chacun est un homme de bonne volonté. Chaque journée comporte labeur et tension. Le personnel s'élève au rang de sur-personnel (Das Persönliche ist aufgehoben im Überpersönlichen) : c'est le point le plus haut de l'existence » (Dasein) ${ }^{6}$.

3 C'est une expérience extraordinaire qui implique la nation allemande entière et la refond en une sorte de corps mystique collectif. L'éclatement de la guerre marque "l'heure de la désindividualisation" (Entselbstung), du ravissement commun dans l'Entier (gemeinsame Entrückung in das Ganze). L'amour ardent pour la communauté (Gemeinschaft) brise les limites du moi. Chacun ne fait qu'un seul rang et un seul corps avec les autres, tous sont unis dans la fraternité, prêts à annuler leur propre moi dans le service " ${ }^{7}$. C'est clairement le langage de l'expérience mystique : "Nous ne sommes plus ce que nous avons été pendant si longtemps : des individus isolés ${ }^{8}$. Et c'est une expérience que Marianne Weber rappelle encore avec émotion bien longtemps après, bien qu'ait disparu en fait le corps mystique de la nation allemande, avant même l'écroulement militaire de l'Allemagne.

4 Des accents mystiques résonnent également dans une lettre de Husserl que nous n'avons à notre disposition que dans la traduction anglaise qu'en a faite son destinataire américain. Encore une fois, l'objet de célébration, c'est l'unité qui s'est forgée au moment de l'éclatement de la guerre, et qui trouve son expression la plus haute dans la communauté des soldats sur le front: "Le sentiment que la mort de 
chacun signifie un sacrifice volontairement offert confère une dignité sublime et hausse la souffrance individuelle dans une sphère qui est au-dessus de toute individualité. Nous ne pouvons plus continuer à vivre comme des personnes privées. L'expérience de chacun concentre en elle-même la vie de la nation entière et cela confère à chaque expérience son moment terrible (tremendous moment). Toutes les tensions, les aspirations passionnées, tous les efforts, toutes les afflictions, toutes les conquêtes et toutes les morts des soldats sur le champ de bataille - tout cela entre collectivement dans les sentiments et dans les souffrances de chacun de nous $»^{9}$.

5 Ce « pathos » de la communauté et de l'Entier ne reste certes pas limité à la culture allemande ou germanique. En ce qui concerne l'Italie, en 1928 encore, dis ans après la fin de la guerre et 6 ans après l'avènement du fascisme au pouvoir, dans le sillage de la guerre, Croce rappellera avec chaleur la " concorde nationale ", l'« état d'âme commun et national » qui préside à l'entrée de l'Italie dans cette « fournaise » qu'est la guerre ${ }^{10}$. En septembre 1917, Croce avait écrit : "Le concept de puissance et de lutte, que Marx avait transporté des États aux classes sociales, semble maintenant revenu des classes aux États ${ }^{11}$. C'est-à-dire que la guerre avait le mérite d'expulser le conflit social de la communauté nationale. C'est en ce sens déjà que s'était exprimé Vilfredo Pareto en 1904 : « S'il y a une grande guerre européenne, le socialisme est repoussé au moins d'un demi-siècle, et la bourgeoisie est sauve pour ce temps-là $»^{12}$. Et au début, cette prophétie semble se réaliser pleinement pour tous les pays participant au conflit. La disparition de «toutes les différences de classe »? Stefan Zweig en parle, comme nous l'avons vu. Mais Marianne Weber fait une observation analogue: «Des hommes innombrables, parmi les simples fils du peuple, n'ont jamais éprouvé tant d'amour qu'ici », dans le lazaret de guerre ${ }^{13}$. Et à son tour une observatrice anglaise (Edith Wharton) décrit ainsi le climat également merveilleux du Paris des derniers jours de juillet 1914: «Il y a seulement deux jours, les Parisiens menaient des milliers d'existences différentes dans la plus complète indifférence ou en plein antagonisme les uns contre les autres, étrangers autant que des ennemis au-delà des frontières... Maintenant ils se rassemblent en foule en s'embrassant, en une aspiration ardente et instinctive à la communauté nationale ", une communauté qui implique et fond en un tout organique même les classes qui, quelques jours avant encore, étaient considérées comme les "classes dangereuses ", et l'étaient effectivement ${ }^{14}$. À ce tableau on peut ajouter le tableau analogue de Curzio Malaparte, toujours en se référant à l'explosion de la guerre : «Cette fois [...] le peuple entier fut appelé à l'aide de la société constituée, ennemie, économiquement et socialement, du peuple [...]. La nation armée, ou plutôt le prolétariat armé, fut le paradoxe de l'année $1914 »^{15}$.

6 De l'Italie qui n'était pas encore entrée en guerre, Croce regarde avec admiration, et peut-être aussi avec un peu d'envie, le spectacle de l'unité nationale de l'Allemagne, où l'enthousiasme et l'effort patriotique se sont communiqués à tout le monde, balayant pour toujours, au moins en apparence, toute trace du conflit social et de la lutte de classe chère à Marx. C'est justement l'Allemagne, où semblait s'être réalisée le plus profondément et sans réserve l'intégration patriotique du mouvement socialiste, qui apparaît au philosophe napolitain comme un modèle chargé d'avenir, qui allait bien audelà des contingences de la guerre : «Tu vois » - écrit-il à un ami le 22 décembre 1914 «je me suis autrefois passionné pour le socialisme à la Marx, puis pour le socialisme 
syndicaliste à la Sorel ; j'ai espéré de l'un et de l'autre une régénération de la vie sociale actuelle. Et les deux fois j'ai vu se dissoudre et s'évanouir cet idéal de travail et de justice. Mais maintenant s'est allumé l'espoir d'un mouvement prolétaire bien encadré et résolu dans la tradition historique, d'un socialisme d'État et de nation; et je pense que cela ne sera pas le fait des démagogues de France, d'Angleterre et d'Italie (qui ouvrent la voie non pas au prolétariat et aux travailleurs, mais, comme le dit mon très cher ami Sorel, aux noceurs), cela sera peut-être le fait de l'Allemagne, qui en donnera l'exemple et le modèle aux autres peuples. C'est pourquoi je juge très différemment des socialistes italiens l'acte accompli par ceux d'Allemagne ; et je crois que ces socialisteslà, qui se sont sentis un tout avec l'État germanique et avec sa discipline de fer, seront les vrais promoteurs de l'avenir de leur classe ${ }^{16}$.

7 Après l'éclatement de la guerre, si on peut encore parler de socialisme, c'est seulement du «socialisme d'État et de nation ». À la fin du XIX ${ }^{e}$ siècle, Engels avait dénoncé le caractère réactionnaire $\mathrm{du}$ " socialisme prussien d'État » (preussischer Staatssozialismus), et - fait encore plus significatif - il avait vu une connexion entre les préparatifs de guerre de Bismarck et le développement de ce «faux socialisme» et "prétendu socialisme $»^{17}$. Maintenant, au contraire, la catégorie et l'expression «socialisme d'État " acquiert une signification positive, et même Croce élabore son modèle en regardant explicitement vers l'Allemagne. Celle-ci, donc, n'est pas du tout isolée dans la célébration d'une communauté baptisée par la guerre ${ }^{18}$.

Certes, la célébration de la Gemeinschaft, opposée à la Gesellschaft, a derrière elle en Allemagne une plus longue histoire. Toutefois il faut faire très attention à distinguer correctement les particularités de la tradition culturelle allemande. On a pu parler de « socialisme de guerre » également à propos de l'Angleterre de $1914-1918^{19}$; Croce ne perçoit aucune contradiction entre ses convictions libérales et sa profession de foi dans le «socialisme d'État et de nation " : l'effort prolongé de mobilisation chorale et totale rend inévitable, dans tous les pays belligérants, même ceux aux traditions libérales les mieux consolidées, le recours à une idéologie de la communauté en mesure d'exiger et de justifier le sacrifice inconditionné de millions de personnes. Ce climat idéologique, seuls continuent à y rester étrangers les marxistes pour ainsi dire irréductibles et les pacifistes. Si bien que les socialistes d'une ville italienne qui, en septembre 1916, refusèrent de participer à l'allégresse générale à l'annonce de la conquête de Gorizia (qui avait coûté tant de sang) sont accusé par Croce d'« insensibilité morale», de " cécité et étroitesse d'esprit ». Ils avaient le tort de s'auto-exclure d'une communauté nationale à la fois morale et spirituelle. Ils oubliaient de toute façon que «maintenant comme par le passé, l'histoire place en premier lieu la Patrie, et la défense de la Patrie, et la gloire de la Patrie, et seulement en deuxième lieu, et à l'intérieur de la Patrie, les oppositions des partis et des classes $»^{20}$. Les socialistes italiens - écrira encore Croce en 1928 - «plaçaient d'autres idéaux au-dessus de la patrie et contre la patrie », et ainsi, "idéalement, se détachèrent du peuple auquel ils appartenaient " ${ }^{21}$.

Quelques années avant la guerre, Croce avait déploré le fait que le mouvement socialiste d'inspiration marxiste ait miné la «conscience de l'unité sociale ». Il en avait dérivé « une décadence générale du sentiment de discipline sociale: les individus ne se sentent plus liés à un grand tout, soumis à celui-ci, coopérant en lui, puisant leur valeur dans le travail qu'ils accomplissent dans le tout ${ }^{22}$. L'explosion de la guerre apparaît à Croce comme l'occasion pour recomposer ce "grand tout", comme le montrait 
l'exemple allemand du «socialisme d'État et de nation », et il était donc indigné par la réticence persistante des socialistes italiens.

Pour employer des catégories du débat politique contemporain, on pourrait dire qu'en ce moment ce sont en premier lieu les marxistes qui s'opposent à l'organicisme et à Pholisme qui, bien que sous des formes différentes, caractérisent Croce en même temps que les idéologues des autres pays belligérants. Il est significatif qu'en 1928, prenant ses distances par rapport à cette Kriegsideologie à laquelle, dix ans auparavant, il avait luimême fourni une contribution importante avec les Betrachtungen eines Unpolitischen, Thomas Mann signale précisément en Marx le critique le plus radial de l'«idée de communauté » (Gemeinschaftsidee), qui en Allemagne se charge de motifs "romanticopopulaires » (volksromantisch), ou bien völkisch, et dont l'antithèse la plus radicale est de toute façon représentée par l'«idée socialiste de société» (sozialistische Gesellschaftsidee) ${ }^{23}$.

11 Thomas Mann semble considérer l'idée de communauté comme quelque chose de typiquement allemand. Nous pouvons dire au contraire qu'au moins en ce qui concerne les années de guerre, des formes d'organicisme communautaire, plus ou moins emphatiques, s'affirment aussi en dehors de l'Allemagne. À l'occasion de la polémique dont nous avons parlé, suscitée par la prise de Gorizia, il est significatif qu'aux accusations, adressées aux socialistes, d'insensibilité pour la Patrie, Gramsci réponde en accusant Croce d'avoir « une idée territoriale de la patrie, de la nation » et de se mettre, en dernière analyse, au même niveau que Maurice Barrés ${ }^{24}$.

Il faut donc essayer de configurer différemment la particularité de l'évolution idéologique allemande. En opposant "idée de communauté " et "idée socialiste de société », Thomas Mann reprend, c'est clair, la distinction formulée en son temps par Tönnies entre Gemeinschaft et Gesellschaft. Cette distinction ou opposition connaît une fortune notoire en Allemagne au cours de la Première guerre mondiale et trouve sa consécration chez Marx Scheler qui, en formulant la « table des catégories de la pensée anglaise ", accuse ce dernier de confondre, entre autres, Gemeinschaft et Gesellschaft ${ }^{25}$. Il est clair alors que la première vient coïncider avec l'Allemagne, la seconde avec ses ennemis. Et voilà donc que le triomphe de la « communauté » forgée par la guerre est vu par de larges secteurs de la culture, de la presse et de l'opinion publique allemandes comme le déclin non seulement du marxisme, mais aussi des "idées de 1789 ", auxquelles on oppose les «idées de $1914 »^{26}$; le « socialisme d'État et de nation », pour utiliser l'expression de Croce, triomphe non seulement sur le socialisme marxiste, mais aussi sur le libéralisme et sur la démocratie. Bien au-delà des vicissitudes et des contingences de la guerre, on dirait que se cristallise l'opposition entre une " communauté » fortement imprégnée - observe Mann - d'éléments " aristocratiques » et «cultuels » (kultisch) et une « société » non seulement démocratique, mais surtout profane, qui trouve son expression la plus accomplie et la plus répugnante dans le socialisme marxiste, étranger, avec le libéralisme et la démocratie et encore plus qu'eux, à l'authentique âme nationale allemande ${ }^{27}$.

13 Mais ce n'est pas seulement pour l'expérience de la « communauté » que la Première guerre mondiale, au moins à ses débuts, apparaît comme "grande et merveilleuse " à des masses considérables d'hommes, à des intellectuels, et à de grands intellectuels 
pourtant si différents entre eux, et à Max Weber lui-même. Ce dernier regrette de ne pouvoir vivre personnellement l'expérience du front, lui qui se considère - c'est ce qu'il écrit à sa mère - "parmi tes fils le plus doué d'un tempérament guerrier ». Malgré ses tragédies et ses horreurs, "cela vaut la peine, de vivre cette guerre; il aurait encore mieux valu être présent, mais hélas je ne puis être utilisé sur le champ de bataille». Et pourtant, même le travail plus ou moins bureaucratique dans un lazaret de l'arrière permet de participer à la richesse et à la plénitude spirituelle du moment : "Même ainsi, la vie produit pourtant toujours des choses qui la rendent digne d'être vécue ${ }^{28}$. Et le lazaret semble, même constituer un poste d'observation privilégié, justement en vertu du drame quotidien qui s'y déroule : "Ici nous avons fourni la preuve que nous sommes un grand peuple de culture (ein großes Kulturvolk) : des hommes qui vivent au beau milieu d'une culture raffinée, et qui, en dehors de leur milieu, montrent qu'ils sont à la hauteur de l'horreur de la guerre (ce n'est certes pas une entreprise pour un nègre du Sénégal!), et qui ensuite malgré tout reviennent avec tant de dignité, comme c'est le cas pour la grande majorité de nos gens: cela, c'est être des hommes authentiques (echtes Menschentum) $»^{29}$. L'expérience et la tension de la guerre semblent même être utiles à la santé de Weber. Après une longue journée de dur labeur, même les heures de repos et de détente semblent tourner autour du thème de la guerre. Marianne nous rapporte ce qui suit: "Souvent il y a des amis du front qui, étant blessés, se trouvent dans leur patrie pour quelques temps. Ils sont au centre de l'attention. Ils ne peuvent s'arrêter de raconter. En chacun d'eux, en effet, les événements se reflètent d'une manière différente; chacun est imprégné du bonheur donné par le don de sa vie et le dévouement à une cause élevée. Il est magnifique, le regard de ces figures sévères. Certains - encore adolescents quelque temps avant apparaissent maintenant en uniforme, comme des hommes. En général, les traits des soldats en congé apparaissent comme singulièrement tendus : ils révèlent une vigilance intérieure constante, une responsabilité sévère et les expériences vécues à proximité de la mort $»^{30}$.

Ici, le thème de la communauté, du "dévouement à une cause élevée ", se fond avec le thème de l'efficacité formatrice et pédagogique de la "proximité de la mort». Naturellement, on peut retrouver ce thème également dans l'idéologie de guerre des pays de l'Entente; toutefois, dans le cadre de la culture germanique, il connaît une diffusion et une épaisseur tout à fait particulières. On dirait que la proximité de la mort arrive maintenant à faire partie intégrante de l'authentique Bildung : un motif classique est repris et interprété dans la nouvelle situation de guerre.

Marianne Weber continue ainsi son évocation des années de guerre : « Le premier Noël de guerre fut profondément imprégné d'amour, de poésie et de solennité ». Max Weber s'adresse aux soldats en congé réunis autour de l'arbre de Noël : « Il sait qu'ils doivent retourner au front. Sa voix est comme une musique d'orgue. Il parle de la grandeur de la mort au combat. Dans la vie quotidienne, la mort nous arrive incomprise, comme un destin contraire à la raison, et dont on ne peut retirer aucun sens. Nous devons seulement la subir. Mais chacun d'entre vous sait pourquoi il meurt, quand le sort le frappe. Celui qui reste sur le champ de bataille est une semence pour l'avenir. La mort héroïque pour la liberté et l'honneur de notre peuple est l'entreprise la plus haute, la plus efficace pour nos enfants et les enfants de nos enfants $»^{31}$. Ce discours n'est semblable qu'en partie aux habituels appels au sacrifice dont est pleine la rhétorique de guerre de tous les pays. Il y a même un élément particulier de la Kriegsphilosophie allemande: le champ de bataille devient le lieu privilégié pour saisir le sens 
authentique de la vie; la proximité de la mort empêche ce refoulement qui est le propre de la vie quotidienne et est donc en mesure de conférer à l'existence humaine une profondeur et une intensité impossibles à atteindre autrement.

Ce sont des thèmes qui apparaissent avec une clarté encore plus grande dans une leçon de Husserl de novembre 1917, dans le cadre de laquelle est examinée l'influence de la guerre ( « cette guerre, ce destin, grand et sévère au-delà de toute imagination, de notre nation allemande ») sur la vie spirituelle : «Idées et idéaux sont de nouveau en marche et trouvent de nouveau un cœur ouvert pour les accueillir. La façon unilatéralement naturaliste de penser et de sentir perd sa force. La détresse et la mort (Not und Tod) sont aujourd'hui les éducateurs. Désormais depuis des années la mort n'est plus un événement exceptionnel, qu'on cache par des conventions fastueuses et solennelles, sous des tas de couronnes de fleurs, et qu'on puisse falsifier dans sa sévère majesté. La mort a de nouveau conquis son droit original sacré. Elle est de nouveau là, rappelant l'attention, dans le temps, sur l'éternité. Et ainsi de nouveaux organes nous ont-ils poussé, pour saisir et comprendre l'idéalisme allemand $\|^{32}$. La guerre tend désormais à se configurer comme une meditatio mortis opportune, salutaire, indispensable ; c'est une sorte d'exercice spirituel, qui permet de sortir de la banalité et de la dispersion de la quotidienneté et de récupérer le sens authentique de la vie.

17 C'est également l'opinion de Simmel et de Scheler : le premier célèbre dans la guerre (et dans la proximité de la mort qu'elle comporte) la "situation absolue» (absolute Situation), qui au-delà de la banalité de la vie quotidienne, du «compromis » et du " point de vue de la quantité » qui la caractérisent, exige la « décision absolue » de la quantité qui la caractérisent, exige la " décision absolue » (absolute Entscheidung), révèle la puissance des «idées", comme "instance suprême ", même à celui qui " n'a jamais

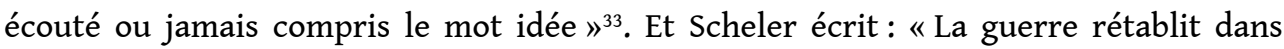
notre conscience le rapport vrai, adéquat à la réalité, entre vie et mort »; la guerre met fin à ce phénomène commun qui consiste à « ne pas voir, et même ne pas vouloir voir la mort ", elle met fin à son "refoulement et à sa dissimulation » par « le voile trompeur d'une praxis vitale devenue obtuse habitude $»^{34}$. La meditatio mortis est encore une fois le résultat de la « métaphysique de la guerre » théorisée par Scheler ${ }^{35}$.

18 Comme preuve du profond enracinement dans la culture germanique du thème de la guerre comme meditatio mortis, on peut proposer l'exemple de Freud, certainement éloigné de la Kriegsphilosophie, et toutefois auteur, en 1915, d'un essai profondément imprégné du débat et de l'esprit du temps. C'est un essai qu'il est donc intéressant de citer assez largement: « Il y a en nous la tendance évidente à écarter la mort, à l'éliminer de la vie. Nous avons essayé d'en faire taire la pensée [...]. Nous insistons en général sur la cause accidentelle de la mort : accident, maladie, infection, âge avancé : révélant ainsi une tendance à abaisser la mort de fait nécessaire à fait casuel ». Ce refoulement de la mort constitue un motif de sécheresse et d'aridité: «La vie s'appauvrit, perd de son intérêt s'il n'est pas permis de risquer ce qui, dans son jeu, est l'enjeu suprême, c'est-à-dire la vie elle-même [...]. La tendance à exclure la mort du grand livre de la vie nous a ainsi imposé beaucoup d'autres renoncements et beaucoup d'autres exclusions. Pourtant, la devise hanséatique disait: Navigare necesse est, vivere non necesse! Naviguer est nécessaire, vivre n'est pas indispensable ! ». La guerre met fin à ce refoulement falsificateur de la vie spirituelle: «Il est clair que la guerre devait balayer cette façon conventionnelle de considérer la mort. La mort ne peut plus, aujourd'hui, être niée; nous sommes contraints à y croire. Les hommes meurent 
vraiment; et non plus un à la fois, mais en grand nombre, souvent par dizaines de milliers chaque jour. Ce n'est plus quelque chose de casuel, désormais [...]. Et la vie est à nouveau devenue intéressante, et elle a retrouvé tout son contenu ${ }^{36}$. Aux yeux de Freud, la guerre apparaît comme le moment de la destruction de l'artifice et du retour à l'authentique: «Elle élimine les sédimentations successives déposées en nous par la civilisation et laisse réapparaître l'homme primitif ». C'est une leçon à ne plus oublier. La guerre ne se laisse pas éliminer. « Le problème qui s'impose alors est le suivant : estce qu'il ne vaudrait pas mieux céder, nous adapter à la guerre ? [...]. Est-ce qu'il ne serait pas préférable de restituer à la mort, dans la réalité et dans notre pensée, la place qui lui revient [...] ? ». Pour Freud il n'y a pas de doute : « Si vis vitam, para mortern. Si tu veux pouvoir supporter la vie, dispose-toi à accepter la mort ${ }^{37}$.

Un cas extraordinaire d'autre part, c'est celui de Wittgenstein. Enrôlé volontaire, lors de l'éclatement du conflit, il va tout de suite au-devant de la déception la plus cuisante. Il se sent entouré par la haine et par la "vulgarité " générale ${ }^{38}$ (la troupe d'origine populaire et enrôlée sur la base de la conscription obligatoire n'avait aucune sympathie à l'égard des volontaires, intellectuels d'extraction bourgeoise); il pense même au suicide ${ }^{39}$. Pourtant, même chez Witgenstein, le contact avec la mort semble jouer un rôle purificateur et pédagogique: "Maintenant j'aurais la possibilité d'être une personne décente, parce que je me trouve face à face avec la mort $»^{40}$; ou encore: "Peut-être la proximité de la mort m'apportera-t-elle la lumière de la vie ${ }^{41}$. Enfin: "Seule la mort donne un sens à la vie ${ }^{42}$.

À son tour également, le Thomas Mann des années de la Première guerre mondiale souligne l'« élévation religieuse, l'approfondissement et l'ennoblissement de l'âme et de l'esprit » qui peuvent être produits par la " proximité quotidienne, prolongée pendant des années, avec la mort ». Et de nouveau revient le thème de la guerre comme élément de la Bildung: "La pauvre épouse du guerrier qui revient à la maison accueillera un homme différent par rapport à celui dont elle a pris congé ». En ce sens, le résultat de la guerre peut bien être une "humanité supérieure" (höhere Menschlichkeit), "une élévation, un accroissement, un ennoblissement de l'humain $»^{43}$. Ce gain spirituel net comporte une attitude à l'égard de la mort telle qu'elle exclut peur et refoulement. Malgré ses horreurs, la guerre peut produire «liberté, une liberté et une sérénité religieuse, une attitude de détachement de la vie, une libération au-delà de la crainte et de l'espoir, qui sans aucun doute signifie le contraire d'une dégradation morale, c'est-àdire le dépassement même de la mort $»^{44}$.

Et c'est dans ce même contexte qu'on peut situer le thème de la "sympathie avec la mort» (Sympathie mit dem Tode), vue comme «la formule et la détermination fondamentale de tout romantisme", et même comme "le dernier mot du romantisme ", mais justement pour cela profondément étrangère à la Zivilisation occidentale, toute entière fondée sur une foi superficielle dans le "progrès ", dans la "raison, dans le bonheur", c'est-à-dire fondée sur le refoulement du négatif de l'existence, coupable, en dernière analyse, de "trahison à l'égard de la Croix » (Verrat am Kreuz) $)^{45}$.

Dans la Kriegsideologie dont, la meditatio mortis est un motif central : elle est considérée comme le propre de la profondeur de l'âme allemande, tandis qu'elle serait étrangère à la superficialité de l'Occident. Une vie dont on aurait refoulé la pensée de la mort observe Freud - «devient vide, insipide comme un flirt américain, dans lequel on sait dès le début qu'il ne doit rien arriver, à la différence d'une relation amoureuse du vieux 
continent, dans laquelle les deux protagonistes sont continuellement conscients des conséquences sérieuses auxquels ils s'exposent $»^{46}$. Ce qui est intéressant, dans cette comparaison, ce n'est pas seulement le rapprochement entre l'intensité de l'expérience vitale de la guerre et de la proximité de la mort, et l'intensité de l'expérience authentiquement érotique; ce qui est encore plus significatif, c'est le fait que le lieu privilégié de l'aplatissement de la vie consécutif au refoulement de la mort est signalé hors du "Continent ", avec une référence explicite à l'Amérique, mais avec une allusion probable également à l'Angleterre, et en opposition, bien sûr, à l'Europe continentale, mais en premier lieu, de façon transparente, à l'Allemagne. À son tour, Husserl fait coïncider, comme nous l'avons vu, le déclin, à la suite de la guerre et de l'expérience de la mort, de la "façon naturaliste et unilatérale de penser et de sentir", avec la renaissance de l'«idéalisme allemand», en particulier de l'idéalisme fichtéen ${ }^{47}$. De la même façon, Simmel, en attribuant à la guerre le mérite d'avoir remis à l'ordre du jour la puissance des "idées", se réclame de $\mathrm{Kant}^{48}$ : de toute façon on voit réapparaître l'opposition de l'Allemagne au matérialisme ou au naturalisme, à la superficiale dépourvue de profondeur spirituelle, métaphysique ou religieuse, propre à ses ennemis. Ce thème trouve d'autre part sa formulation la plus vulgaire dans l'antithèse chère à Sombart entre « héros » allemands et « marchands », surtout anglais.

À la lumière de tout cela, on peut comprendre le jugement exprimé sur l'Allemagne, dans la période entre les deux guerres, par l'homme d'État français Clemenceau : « C'est le propre des hommes que d'aimer la vie. Les Allemands n'ont pas cette impulsion [...]. $\mathrm{Au}$ contraire, ils sont remplis d'une nostalgie morbide et satanique de la mort. Comme ils aiment la mort, ces hommes! Frémissants, comme en état d'ivresse et avec un sourire d'extase, ils regardent vers elle comme vers une sorte de divinité [...]. Même la guerre est pour eux un pacte avec la mort $»^{49}$. La configuration de son âme et de son essence, à laquelle l'Allemagne procédait, avait fini par faire école également chez ses ennemis, naturellement avec un jugement de valeur renversé. Dans un cas comme dans l'autre, une tendance historique réelle, ou l'idéologie dominante à un moment déterminant, se raidit en un stéréotype national immobile qui laisse peu d'espace à des exceptions et à des variations. Il ne faut pas oublier que cette célébration de la guerre et de la proximité de la mort comme une sorte d'exercice spirituel rencontre des résistances aussi en Allemagne et dans l'espace culturel de langue allemande. Parmi les auteurs mêmes que nous avons cités ne manquent pas ceux qui prennent leurs distances par rapport aux positions exprimées au cours de la Première guerre mondiale. Wittgenstein n'attend même pas la fin du conflit pour se rendre compte de la guerre, loin de représenter un moment de maturation spirituelle, par la meditatio mortis, signifie en réalité « la victoire complète du matérialisme et le déclin de toute sensibilité au bien et au mal $»^{50}$. C'est un jugement qu'on peut rapprocher de la définition que donne Lukâcs de son temps, comme l'époque de la "peccaminosité accomplie " ${ }^{51}$; par rapport à la célébration, spiritualiste et édifiante, qui de la guerre et de la proximité de la mort, la définition de Lukâcs (et aussi celle de Wittgenstein) représente l'antithèse la plus radicale, même s'il s'agit d'une antithèse formulée elle-même dans un langage spiritualiste. Mais ce n'est pas un hasard si Marianne Weber, bien plus représentative des orientations de l'intellectualité allemande, reproche à Lukâcs une sorte d'insensibilité congénitale à la « grandeur » de la guerre ${ }^{52}$. 
Il reste donc le fait que la "métaphysique de la guerre »- pour employer l'expression de Scheler - réussit à imprégner d'une façon on ne peut plus capillaire les milieux culturels les plus divers, et il reste tout autant le fait que cette métaphysique fait levier non seulement sur le thème de la « communauté ", mais aussi sur celui de la « mort ». L'entrelacement de ces deux thèmes est analysé avec une grande lucidité par Max Weber : « La guerre, comme réalisation de la menace de violence, crée vraiment dans les communautés politiques modernes un pathos, un sentiment communautaire (Gemeinschaftsgefühl), suscitant ainsi un dévouement et une communauté du sacrifice inconditionné parmi les combattants (bedingungslose Opfergemeinschaft der Kämpfenden) [...]. Et de plus, la guerre donne au guerrier quelque chose d'unique dans sa signification concrète: le sentiment d'un sens et d'une consécration de la mort, qui appartient seulement à la mort en guerre. La communauté de l'armée sur le champ de bataille est aujourd'hui, comme à l'époque de l'ancienne communauté germanique (Gefolgschaft), une communauté jusqu'à la mort (Gemeinschaft bis zum Tode). La mort sur le champ de bataille se distingue de cette mort qui n'est que le sort commun des hommes, un destin qui atteint chacun de nous sans qu'on puisse jamais dire pourquoi il a échu précisément à cette personne et précisément à ce moment-là [...]. À la différence de cette mort qui n'est qu'un fait inévitable, dans la mort sur le champ de bataille, et seulement là, dans une mesure aussi massive, l'individu peut croire qu'il sait qu'il meurt pour quelque chose $"^{53}$. C'est une analyse qui met à profit l'expérience du conflit mondial, mais qui ne semble pas se traduire en une critique de la Kriegsideologie. Et même, quand nous lisons quelle signification la guerre est en mesure de donner à la mort de l'individu, nous ne pouvons ne pas penser aux paroles que Weber adressait, à l'occasion de Noël 1914, selon le témoignage de sa femme, aux soldats qui s'apprêtaient à retourner au front. Certes, dans le texte de la Religiossoziologie, il est souligné que la signification de la mort en guerre est d'objet non d'un savoir, mais d'une croyance; mais l'homme politique, animé d'une passion nationale et nationaliste, peut bien aller au-delà des précautions du savant fidèle au critère de la Wertfreiheit. Et quand Weber, toujours dans le même contexte, ajoute que la guerre suscite, à l'égard des "nécessiteux", un sentiment de solidarité qui «brise toutes les barrières des associations naturelles" (naturgegebene Verbãnde) ${ }^{54}$, force nous est de penser à l'observation déjà citée de Marianne Weber à propos du lazaret, où les soldats des classes populaires jouissaient de soins et d'attentions chaleureuses dont ils n'avaient jamais été l'objet précédemment.

L'entrelacement du thème de la « communauté » et du thème de la " mort » produit en Allemagne un mélange idéologique particulièrement trouble et explosif. Dans ce contexte, la figure centrale est sans aucun doute constituée par Ernst Jünger. Dans son œuvre est radicalisé et répété en des variations infinies le thème de la « proximité de la mort ", qui rend la vie, il est vrai, "plus douloureuse », mais aussi "plus douce »; le «baptême du feu » devient une véritable fête et une source d'ivresse ${ }^{55}$. Il n'y a pas de doute : la vie est plus intense, plus riche, plus scintillante, précisément là où la mort fait fureur ${ }^{56}$. Le jugement de Clemenceau, dont nous avons parlé, apparaît comme un diagnostic lucide et rigoureux, lorsqu'on l'applique à Ernst Jünger. Oui, la mort s'élève au rang de véritable divinité et le rite sacré qui se déroule en son honneur produit non seulement l'ivresse, mais jusqu'à l'« extase »; "cette condition, qui est le propre du 
saint, du grand poète et du grand amour, est accordée aussi au grand courage »; le guerrier « est fondu dans le tout et court vers les portes obscures de la mort comme le projectile vers sa cible $»^{57}$. Surtout, la participation à ce rite sacré devient la condition inéliminable pour participer à la communauté authentique. Au front, « un unique grand destin nous soulève sur la même vague. Là nous avons été pour une fois ensemble comme organisme (Organismus) face au monde extérieur hostile, comme des hommes qui, malgré leurs petits problèmes, souffrances et joies, sont soudés ensemble par un devoir supérieur $»^{58}$.

Nous avons vu que le Thomas Mann d'après-guerre identifie dans la "communauté cultuelle» (kultische Gemeinschaft) l'élément caractéristique de l'idéologie allemande. Maintenant, chez Ernst Jünger, le culte tourne clairement autour de la mort, du sang. On comprend alors comment la communauté - objet de célébration - devient en premier lieu la " communauté guerrière $~_{59}$, et cette kriegerische Gemeinschaft tend à son tour à se configurer comme "communauté de sang" (Blutgemeinschaft ${ }^{60}$ ), une communauté baptisée par le sang versé en guerre, et qui prend facilement, même si ce n'est pas nécessairement, des aspects raciaux ou racistes.

Arrivé à ce point, le nazisme peut bien assumer l'héritage de la Kriegsideologie. En 1934, Goebbels célèbre les combattants allemands qui «rapportèrent des tranchées une nouvelle façon de penser. Dans les terribles sacrifices et dans les dangers ils avaient vécu un nouveau type de communauté, qu'ils n'auraient jamais pu expérimenter dans des périodes de bonheur »; de l'expérience de la mort et de l'« égalité » devant la mort jaillit l'engagement solennel à ne pas tolérer que s'ouvre, surtout "en temps de danger ", une brèche au sein du peuple ${ }^{61}$.

La célébration de l'efficacité formatrice de la proximité de la mort comporte un nouveau chef d'accusation contre la Gesellschaft: celle-ci est synonyme de banale recherche de la sécurité et de la tranquillité propre au monde bourgeois (dans lequel sont aussi pleinement insérés marxisme et mouvement ouvrier) ${ }^{62}$. Le thème cher à Sombart réapparaît, celui de l'affrontement entre « héros » et «marchands ». C'est un thème qui avait trouvé dans les années de la guerre, bien que dépouillé de toute exaspération vitaliste et guerrière, un certain écho même chez Thomas Mann: l'« humain » serait incomplet si la figure du « guerrier » (Krieger) venait à manquer, s'il fallait choisir seulement entre "marchands et lettrés " ${ }^{63}$. Il condamnait ainsi l'« État bourgeois de la sécurité, l'état-parapluie » qui prétendait bannir pour toujours de la vie le « terrible » (das Furchtbare) et l'« élémentaire » (das Elementare) en instaurant "sur la terre la verte et générale félicité du pâturage ", et donc "sécurité, absence de dangers, confort et facilité de la vie» (Sicherheit, Ungefährlichkeit, Behagen, Leichtigkeit des Lebens) ${ }^{64}$. D'ailleurs, il y a déjà dans le texte de 1918 des accents sensiblement différents et même contradictoires par rapport à ceux que nous venons de voir (qu'on pense à la célébration de la Bürgerlichkeit, même en opposition à la politisation démocratique ${ }^{65}$ ). Et de toute façon, Thomas Mann prend clairement ses distances par rapport à la Kriegsphilosophie dans les années de la République de Weimar, critiquant avec force la rhétorique irrationaliste de la «vie » qui avait contribué à précipiter l'Allemagne dans la guerre ${ }^{66}$.

29 Mais ce bilan autocritique de la Première guerre mondiale ne connaît qu'une maigre diffusion dans les milieux intellectuels allemands. Comme Sombart, Jünger parle du dégoût qu'éprouve le "guerrier né » pour la "vie des boutiquiers ", à l'enseigne de la " sécurité », caractéristique de l'Occident et de la France en particulier ${ }^{67}$. La Première 
guerre mondiale, bien loin de constituer une catastrophe ou même seulement un accident de parcours, est saluée par Jünger comme la fin de l'époque bourgeoise qui prétendait établir la « sécurité » et bannir le « caractère dangereux » (das Gefährliche) ${ }^{68}$. C'est en des termes analogues que s'exprime Spengler l'année suivante: la vie «sans danger, dans un confort tranquille, est ennuyeuse et sénile ». « La vile sécurité du siècle dernier est finie. La vie dans le danger, la vie authentique dans l'histoire, rentre dans ses droits » ${ }^{69}$. Et Jünger écrit : «Le danger [...] domine maintenant le présent»; on assiste ajoute-t-il, à " un nouveau mariage de la vie avec le danger ", tandis que ceux qui s'obstinent à regarder avec nostalgie vers la sécurité perdue appartiennent à la "race des vaincus " $^{70}$; on a désormais définitivement réfuté «l'utopie de la sécurité bourgeoise $»^{71}$, et l'« irruption de l'élémentaire dans l'espace vital» est irrévocable ${ }^{72}$. Jünger va jusqu'à affirmer, comme on le sait, qu'« il est infiniment préférable d'être criminel plutôt que bourgeois $\aleph^{73}$. Parvenu à ce point de radicalisation, cet ultérieur motif de la Kriegsphilosophie (la rhétorique de la vie dangereuse) est mûr lui aussi pour être reçu en héritage par le nazisme, d'autant plus que Jünger identifie la Gesellschaft détestée à la banale et philistine République de Weimar, arrivée au pouvoir par une trahison consommée aux dépens du " guerrier allemand $»^{74}$.

La rhétorique du «vivre dangereusement » culmine dans l'évocation du «destin »: le Schicksal est pour Spengler le contraire de la "vile sécurité » il est synonyme de " conception tragique de la vie " $^{75}$. C'est en des termes analogues que s'exprime Jünger : les temps demandent du courage et « avoir du courage signifie être à la hauteur de tout destin $»^{76}$. La guerre elle-même est un destin, et le peuple allemand a dû selon Weber, "obéir à ce destin", indépendamment de tout calcul sur l'issue du conflit, pour défendre, comme nous le savons, son " honneur " ${ }^{77}$. Nous sommes-là en présence d'un mot-clef de la Kriegsphilosophie, et même de celui qui semble résumer et unifier tous les autres. Au début du conflit, Thomas Mann oppose Voltaire à Frédéric II, pris respectivement comme symbole de la France et de l'Allemagne, le premier comme le représentant de la «clarté sèche » et le second comme celui du « destin enveloppé de nuées $»^{78}$.

31 Le terme en question, outre la signification déjà signalée, en a d'autres, multiples, auxquelles nous pouvons ici seulement faire allusion. Il signifie la transcendance, rendue plus que jamais évidente par la guerre, de la communauté par rapport à l'individu. "La femme est enracinée dans le terrain du destin allemand (deutsches Schicksal), son sort aussi (Geschick) dépend de la fin de cette guerre $»^{79}$. Si Weber pense en premier lieu à la situation de guerre, Jünger procède à une opposition générale et radicale entre sociétés fondées sur un « rapport contractuel et révocable » et sociétés fondées sur le « destin » (Schicksal), dont les liens sont donc indissolubles pour la vie et pour la mort ${ }^{80}$. En ce sens, le "pathos" du destin se soude étroitement avec le "pathos » de la Gemeinschaft, comme avec celui du danger et de la mort. Nous pouvons alors mieux comprendre pourquoi, aux yeux de Weber, l'Amérique est dépourvue de "destin historique » et, aux yeux de Spengler, il manque, aussi bien à l'Amérique qu'à l'U.R.S.S., « l'élément de la tragicité historique authentique, le grand destin qui pendant des siècles a éduqué et rendu profonde l'âme des peuples occidentaux " ${ }^{81}$, et en premier lieu l'âme allemande. Le "destin", c'est l'histoire séculaire, millénaire de défis au danger et à la mort de la part d'une communauté solidaire grâce à un lien idéal intime, et parfois, dans les versions les plus radicales de cette idéologie, par un lien de sang. 


\section{NOTES}

1. - W. Sombart, Händler und Helden. Patriotische Gesinnungen, München-Leipzig, Duncker \& Humblot, 1915, p. 6.

2. - In E.J. Leed, No Man's Land. Combat and Identity in World War I, Cambridge, 1979, tr. it. Bologna, II Mulino, 1985, p. 63.

3. - M. Weber, Deutschlands weltpolitische Lage, 27/X/1916, in Zur Politik im Weltkrieg, Schriften und Reden 1914-1918, par W.J. Mommsen en collaboration avec G. Hüdinger, Studienausgabe, Tübingen, Mohr (Siebeck), 1988, p. 341-342.

4. - M. Weber, An der Schwelle des dritten Kriegsjahres, 1/VIII/1916, in Zur Politik im Weltkrieg, op. cit., p. 334.

5. - Reporté in Marianne Weber, Max Weber. Ein Lebensbild, Tübingen, Mohr (Siebeck), p. 527, p. 530 et p. 536.

6. - Ibid., p. 529.

7. - Ibid., p. 526.

8. - Ibid., p. 536.

9. - Cette lettre est maintenant reportée dans le vol. XXV de Husserliana : E. Husserl, Aufsätze und Vorträge (1911-1921), par Th. Nenon et H.R. Sepp, L'Aya, Nijhoff, 1987, p. 293.

10. - B. Croce, Storia d'ltalia dal 1871 al 1915, Bari, Laterza (1928), 1967, p. 268-271.

11. - B. Croce, Materialismo storico edeconomia marxistica, Roma-Bari, Laterza, 1973, p. XIV (Préface à la troisième édition).

12. - In M. Isnenghi, // mito délia grande guerra, Bari, Laterza, 1970, p. 8.

13. - Marianne Weber, op. cit., p. 529.

14. - In E.J. Leed, No man's Land, tr. it. cit., p. 65.

15. - C. Malaparte, La rivolta deisanti maledetti, 1919-1920, reporté in M. Isnenghi, op. cit., p. 152.

16. - Maintenant in B. Croce, L'Italia dal 1914 al 1918. Pagine sulla guerra, Bari, Laterza, 1950 3, p. 22.

17. - K. Marx-F. Engels, Werke, Berlin, Dietz, 1955 sqq., vol. XXI, p. 180 ; vol. 20, p. 259 note et vol. XXXV, p. 170 ; à ce propos cf. D. Losurdo, Tra Hegel e Bismarck. La rivoluzione del 1848 e la crisi délia cultura tedesca, Roma, Editori Riuniti, 1983, p. 332-335.

18. - Sur le «socialisme d'Etat» et de guerre en Allemagne, dans les années aux environs du premier conflit mondial, cf. D. Losurdo, La catastrofe délia Germania e l'immagine di Hegel, Milano, Guerini e Associati, 1987, p. 74-78 et p. 105-108.

19. - Cf. A.J.P. Taylor, English History 1914-1945, Oxford, Unive-sity Press 1965, tr. it. Roma-Bari, Laterza, 1975, p. 95-97.

20. - Maintenant in B. Croce, L'Italia dal 1914 al 1918, op. cit., p. 151.

21. - B. Croce, Storia d'ltalia dal 1871 al 1915, op. cit., p. 261 et p. 263.

22. - B. Croce, // partito corne giudizio e corne pregiudizio (1912) et Fede e programmi (1911), in Cultura e vita morale (1914), Bari, Laterza, 1955, p. 195-196 et p. 162-163.

23. - Th. Mann, Kultur und Sozialismus, in Essays, vol. II, par H. Kurzke, Frankfurt a. M. Fischer, 1986, p. 99-101.

24. - A. Gramsci, L'idea territoriale, 1916, in Cronache torinesi, par S. Caprioglio, Torino, Einaudi, 1980, p. 608-609.

25. - M. Scheler, Der Genius des Krieges und der Deutsche Krieg, 1915, in Gesammekte Werke, Band 4, Politisch-pädagogische Schriften, par M.S. Frings, Bern und München, Francke, 1982, p. 249-250.

26. - A ce propos cf. D. Losurdo, La catastrophe délia Germania e Vimmagine di Hegel, op. cit., p. 39 sqq.

27. - Th. Mann, Kultur und Sozialismus, op. cit., p. 98-100.

28. - Reporté in Marianne Weber, op. cit., p. 527 et 530. 
29. - Ibid., p. 531.

30. - Ibid., p. 534.

31. - Marianne Weber, op. cit., p. 535-536.

32. - E, Husserl, Aufsätze und Vorträge (1911-1921), op. cit., p. 269.

33. - G. Simmel, Der Krieg und die geistigen Entscheidungen, München und Leipzig, Duncker \& Humblot, 1917, p. 20.

34. - M. Scheler, Der Genius des Krieges..., op. cit., p. 82-83.

35. - Ibid., p. 79.

36. - S. Freud, Zeitgemässes über Krieg und Tod, 1915, tr. id. in Opère, par CL. Musatti, vol. VIII, Torino, Boringhieri, 1976, p. 137-139.

37. - Ibid., p. 147-148.

38. - L. Wittgenstein, Diari segreti, par F. Funtö, tr. it. Roma-Bari, Laterza, 1987, p. 111 et p. 118.

39. - Ibid., p. 109.

40. - Ibid., p. 58.

41. - Ibid., p. 112.

42. - Ibid., p. 114.

43. - Th. Mann, Betrachtungen eines Unpolitischen (1918), par H. Helbling, Frankfurt a ; M. Fischer, 1988, p. 452-453.

44. - Ibid., p. 450.

45. - Ibid., p. 415-419. Comme le remarque Thomas Mann lui-même, dans ces mêmes pages, le thème de la «sympathie avec la mort » est un «thème constitutif de La Montagne Magique, qui ne verra d'ailleurs so achèvement et sa publication qu'après la fin de la guerre (cf. Der Zauberberg, in Th. Mann, Gesammelte Werke in zwölf Bänden, Frankfurt a.M., Fischer, 1960, vol. III, p. 906). Cf. également Th. Mann, Palestrina, 1917, in Essays, op. cit., vol. III, p. 56-58.

46. - S. Freud, op. cit., p. 138.

47. - E. Husserl, Aufsätze und Vorträge (1811-1921), op. cit., p. 269.

48. - G. Simmel, Der Krieg und die geistigen Entscheidungen, op. cit., p. 20.

49. - Weitere Unterhaltungen Clemenceaus mit J. Martet, Berlin, 1930, p. 54 sqq. (reporté in K. Löwith, Mein Leben in Deutschland vor und nach 1933. Ein Bericht, Stuttgart, Metzler, 1986, p. 141 note 13).

50. - Lettre du 12/IV/1917, citée d'après I. Valent, Invito alpensiero in Wittgenstein, Milano, Mursia, 1989, p. 17-18.

51. - C'est la définition qui apparaît en conclusion de la Théorie du Roman, publié auparavant, dans une revue, puis en 1920, sous forme de livre. Revenant par la mémoire au livre et à l'époque, Lukâcs observera ensuite: "L'époque de la "peccaminosité accomplie" de Fichte signifie que l'Europe, de cette consolidation apparente où les hommes ont vécu jusqu'en 1914, s'est précipitée là où elle se trouve "; cf. Gelebtes Leben, interview de Istvan Eörsi, tr. it. Roma, Editori Riuniti, 1983, p. 59.

52. - Cf. G. Lukâcs, Gelebtes Leben, tr. cit., p. 54.

53. - M. Weber, Gesammelte Aufsätze zur Religionsoziologie (1920), Tübingen, Mohr (Siebeck), 1972, p. 548.

54. - Ibidem.

55. - E. Jünger, Feuer und Blut, 1925, in Sämtliche Werke, Stuttgart, Klett-Kotta, 1978 sq., vol. I, p. 444.

56. - E. Jünger, Der Kampf als inneres Erlebnis, 1922, in Sämtliche Werke, op. cit., vol. VII, p. 36.

57. - Ibid., p. 54.

58. - Ibid., p. 85.

59. - E. Jünger, Feuer und Blut, op. cit., p. 452.

60. - L'expression est de Friedrich Georg Jünger (frère de Ernst): cf. Der Aufmarsch des Nationalismus, Leipzig, 1926, p. 21 (reporté in K. Sontheimer, Antidemokratisches Denken in der Weimarer Republik, München Nymphenburger Verlagshandlung, 1968, p. 57). 
61. - J. Goebbels, Wesen und Gestalt des Nationalsozialismus, Berlin, Junker und Dünnhaupt, 1934, p. 14 et p. 21.

62. - E. Jünger, Der Arbeiter (1932), Stuttgart, Klett-Kotta, Bibliothek der Moderne, 1982, p. 19 et p. 26-27.

63. - Th. Mann, Betrachtungen eines Unpolitischen, op. cit., p. 456.

64. - Ibid., p. 455-456 et 484.

65. - Ibid., p. 94 sqq.

66. - Th. Mann, Deutsche Ansprache. Ein Appell an die Vernunft (1930), in Essays, op. cit., vol. II, p. 115.

67. - E. Jünger, Der Arbeiter, op. cit., p. 55 et p. 57 note.

68. - Ibid., p. 19.

69. - O. Spengler, Jahre der Entscheidung, München, Beck, 1933, p. 10 et p. 12.

70. - E. Jünger, Der Arbeiter, op. cit., p. 58-59.

71. - Ibid., p. 266.

72. - Ibid., p. 160.

73. - Ibid., p. 27.

74. - Ibid., p. 26 ; Il en est de même pour : C. Schmitt, Staatsgefüge und Zusammenbruch des zweiten Reiches. Der Sieg des Bürgers über den Soldaten, Hamburg, Hanseatische Verlagsanstalt, 1934 (voir en particulier, p. 42) l'écroulement de l'Allemagne dans la Première guerre mondiale et l'avènement de la République de Weimar coïncident avec la défaite du «soldat» provoquée par le «bourgeois » (Bürger) imprégné des idées de l'ennemi et donc, au moins objectivement, traître.

75. - O. Spengler, Jahre der Entscheidung, op. cit., p. 12-13.

76. - E. Jünger, Der Kampf als inneres Erlebnis, op. cit., p. 51.

77. - M. Weber, Deutschlands weltpolitische Lage, op. cit., p. 342.

78. - Th. Mann, Gedanken im Kriege, 1914, in Essays, op. cit., vol. II, p. 28.

79. - M. Weber, An der Schwelle des dritten Kr egs jähr es, op. cit., p. 338.

80. - E. Jünger, Der Arbeiter, op. cit., p. 25.

81. - A ce propos, cf. D. Losurdo, Tramonto e trasfigurazione delvoccidente. Heidegger e la filosofia tedesca tra le due guerre, in G.M. Cazzaniga, D. Losurdo, L. Sichirollo, Tramonto dell'Occidente? Urbino, Quattro Vend, Istituto Italiano per gli Studi Filosofici, 1989, p. 132 sqq.

\section{RÉSUMÉS}

Le début de la Première guerre mondiale est ressenti par d'assez nombreux intellectuels européens, et surtout allemands, comme une nouvelle preuve de la crise irréversible non seulement du matérialisme historique, mais aussi de toute "manière de sentir et de penser de façon exclusivement naturaliste" (Husserl). Il s'agit d'un conflit pour lequel il n'y a pas d'explication économique ou utilitariste, et cela d'autant plus qu'il semble se dessiner en Allemagne, à l'heure du danger, une communauté organique dont Tönnies avait déjà esquissé la théorie, et qui dépasse tous les intérêts et toutes les différences de classes. Il semble par ailleurs qu'on assiste également dans un autre sens à un renouveau du spiritualisme: la guerre et l'expérience du danger rendent impossible de refouler l'idée de la mort, comme cela se fait de façon tout à fait typique dans la réalité quotidienne. La vie dans les tranchées et au front fait donc naître une communauté qui considère avec mépris la bourgeoisie et sa «lâche » exigence de se soustraire au danger et au « destin ». Nous retrouvons ces thèmes de façon explicite chez des 
auteurs comme Sombart et Jünger, mais aussi chez Simmel, Scheler, Weber, Freud et chez le jeune Thomas Mann, ce dernier adaptent cependant par la suite une attitude explicitement critique dans son analyse de cette idéologie de genre.

Der Ausbruch des Ersten Weltkriegs wird von nicht wenigen europäischen und vor allem deutschen Intellektuellen als erneuter Beweis für die irreversible Krise nicht nur des historischen Materialismus, sondern auch einer jeden «einseitigen naturalistischen Denk- und Fühlweise» (Husserl) erlebt. Es handelt sich um einen Konflikt, der nicht auf eine ökonomische oder utilitaristische Erklärung zurückgäführt werden kann, und das um so mehr, als sich in Deutschland in der Stunde der Gefahr die schon von Tönnies theoretisierte organische Gemeinschaft herauszubilden scheint, die über jedes Klasseninteresse und über alle Klassenunterschiede hinausgeht. Auch in einem anderen Sinn scheint man einer Art Wiederaufleben des Spiritualismus beizuwohnen: der Krieg und das Erlebnis der Gefahr lassen die Verdrängung des Todes, die typisch für die Alltäglichkeit ist, unmöglich werden; das Leben in den Schützengräben und an der Front hebt daher eine Gemeinschaft aus der Taufe, die voller Verachtung auf das Philistertum und dessen «feigen» Anspruch blickt, der Gefanr und dem «Schicksal» auszuweichen. Diese Themen finden wir ausführlich bei Autoren wie Sombart und Jünger, aber auch bei Simmel, Scheler, Weber, Freud und beim jungen Thomas Mann behandelt, wobei letzterer jedoch in der Folgezeit $\mathrm{zu}$ einer eindeutig kritischen Analyse dieser Kriegsideologie übergeht. 